EPJ Web of Conferences 71, 00091 (2014)

DOI: 10.1051/epjconf/20147100091

(C) Owned by the authors, published by EDP Sciences, 2014

\title{
Open heavy flavour at the CERN-LHC
}

\author{
André Mischke $e^{1,2, a}$ \\ ${ }^{1}$ ERC-Research Group QGP-ALICE, Institute for Subatomic Physics, Faculty of Science, Utrecht University, \\ Princetonplein 5, 3584 CS Utrecht, the Netherlands \\ ${ }^{2}$ National Institute for Subatomic Physics, Science Park 105, 1098 XG Amsterdam, the Netherlands
}

\begin{abstract}
In this contribution recent open heavy flavour measurements from the CERNLarge Hadron Collider are presented and discussed. The focus is on prompt D meson and heavy flavour decay lepton production in $\mathrm{pp}, \mathrm{p}-\mathrm{Pb}$ and $\mathrm{Pb}-\mathrm{Pb}$ collisions. Open beauty is studied through a displaced $\mathrm{J} / \Psi$ analysis. Heavy quarks allow to probe the degree of thermalization and energy loss mechanism(s) in the hot QCD matter produced in heavy ion collisions, whereas pp provide important baseline measurements for the studies in heavy ion collisions. The $\mathrm{p}-\mathrm{Pb}$ data give an access to investigate cold nuclear matter effects.
\end{abstract}

\section{Introduction}

Quantum Chromodynamics (QCD) is well established as the fundamental theory describing the most powerful force of nature, the strong interaction between quarks and gluons. These quarks and gluons are the building blocks of atomic nuclei and account for over $99 \%$ of the visible mass of the universe. QCD successfully explains a wide range of phenomena, from the mass spectrum of hadrons to deepinelastic scattering processes. One of the characteristic features of QCD is asymptotic freedom where the interaction becomes weak at large momentum transfer, in other words, when the colour charged quarks come close to each other. In contrast, the potential between the quarks increases steeply when they are separated. In fact, the binding force becomes so strong that, in our normal world, the quarks and gluons are permanently confined inside hadrons.

A fascinating and direct consequence of asymptotic freedom is that under the conditions of sufficiently high temperature or density the strongly interacting quarks and gluons are liberated from their hadronic confinement. This extraordinary new state of matter, where the basic degrees of freedom are released, is called the Quark-Gluon Plasma (QGP). In cosmology, it is believed that the early expanding universe consisted of such plasma a few microseconds after the Big Bang. This plasma subsequently underwent a phase transition where quarks and gluons became confined to form colourless hadrons, which represent observed particles today. Quark matter may still exist in the core of neutron stars, where the density could exceed the critical value of the phase transition. The properties of such matter are fundamental predictions of QCD and its study is one of the leading and most active fields in contemporary subatomic physics.

\footnotetext{
ae-mail: a.mischke@uu.nl
}

This is an Open Access article distributed under the terms of the Creative Commons Attribution License 2.0, which permits unrestricted use, distribution, and reproduction in any medium, provided the original work is properly cited. 
The aim of ultra-relativistic heavy-ion physics is to create hot QCD matter and carefully study its properties under controlled laboratory conditions [1,2]. A description of the Large Hadron Collider and the setup and performance of the ALICE, ATLAS and CMS experiments can be found in [3]. The dissociation of quarkonium states (hidden charm and beauty) due to colour screening in the QGP is one of the classic signatures of deconfinement and was discussed at this conference by [4].

\subsection{Jet quenching}

At the onset of the collision, the quark and gluon constituents of the incoming nuclei can undergo hard collisions. In elementary proton-proton interactions these hard-scattered quarks or gluons fragment in the vacuum into jets of collimated, high transverse momentum particles, whose properties can be measured in the detectors. In heavy ion collisions however, these very energetic quarks or gluons traverse through the formed QGP matter and get slowed down or absorbed, much like X-rays traversing a tissue sample. The properties of the hot QCD matter can be studied through the attenuation of these energetic jets [5].

Measurements at the RHIC particle accelerator have revealed a softening and broadening of jets inside the medium [6]. The observed jet attenuation is evidence of the extreme energy loss of quarks or gluons traversing a large density of colour charges (so-called jet-quenching) and reflects the extreme opacity of the medium. First attempts have been made to determine the stopping power of the QGP matter.

The Large Hadron Collider (LHC) currently provides lead-lead collisions at about 14 times higher centre-of-mass energy per nucleon-nucleon pair $\left(\sqrt{s_{\mathrm{NN}}}=2.76 \mathrm{TeV}\right)$. The initial energy density in the collision zone is about a factor of 3 higher than at the RHIC facility. The higher energy density allows thermal equilibrium to be reached more quickly and to create a relatively long-lived QGP phase. Therefore, most of the in-medium effects should be enhanced, and this has already been observed at the LHC. Precise studies of the jet modification in the QGP matter were performed and important aspects of the jet-quenching theory have been tested over a much broader dynamic range than before $[1,2]$.

\subsection{Heavy quarks and parton energy loss}

Of particular interest is the dependence of the parton energy loss on the colour charge and quark mass, which in term allows to gain more information about the transport properties of the QCD matter. Due to their large mass $\left(>1.3 \mathrm{GeV} / c^{2}\right.$ ), heavy quarks (charm and beauty) are formed predominantly in the earlier stage of the collision by initial hard scatterings through gluon fusion processes. Charm quarks are about 250 times heavier than the light up and down quarks that dominate the QGP matter and their mass is not affected by chiral symmetry breaking [7]. Therefore, they allow to test QCD at transition from perturbative to non-perturbative regime. The formation time $t_{\mathrm{f}}$ for charm quarks is around $0.1 \mathrm{fm} / c$ and $0.02 \mathrm{fm} / c$ for beauty quarks $\left(t_{\mathrm{f}} \simeq 1 / 2 m_{\mathrm{q}}\right)$. At the energy of the LHC the expected lifetime of the formed QGP is in the order of $10 \mathrm{fm} / c$. RHIC measurements have shown [6] that heavyquark production by initial state gluon fusion also dominates in heavy ion collisions where many (in part overlapping) nucleon-nucleon collisions occur. Thermal production later in the collision might contribute to heavy-quark production at low transverse momentum [8].

Heavy quarks are ideal probes for the QGP since they experience the full space-time evolution of the expanding system. They travel through the medium and lose energy due to gluon radiation and 



Figure 1. Left panel: Total nucleon-nucleon charm production cross section versus collision energy $[9,10]$. The NLO MNR calculation [13] (and its uncertainties) is shown by solid (dashed) lines. Right panel: $\mathrm{D}^{*+}$ jet production rate $R\left(p_{\mathrm{T}}, z\right) / \Delta z$ in the jet $p_{\mathrm{T}}$ range $25-30 \mathrm{GeV}$ versus $z$, measured in $7 \mathrm{TeV}$ pp interactions by ATLAS [14]. The data are compared with predictions of the Monte Carlo event generators PYTHIA, HERWIG, POWHEG+PYTHIA and POWHEG+HERWIG. The inset shows the ratio of the measurement to the POWHEG+PYTHIA prediction.

multiple collisions. Beauty quarks are even three to four times heavier than charm quarks (but much less abundant). These large masses mean that charm and beauty quarks have much higher penetrating power than light quarks and gluons.

\subsection{Initial- and final-state effects}

The particle production yield in heavy ion collision has contributions from initial- and final-state effects. They can be quantified using the nuclear modification factor $R_{\mathrm{AA}}$, where the particle yield in heavy ion collisions is divided by the yield in proton-proton reactions scaled by the number of binary collisions. $R_{\mathrm{AA}}=1$ would indicate that nucleus-nucleus collisions can be considered as an incoherent superposition of nucleon-nucleon interactions. Initial-state effects, such as Cronin enhancement, nuclear shadowing and gluon saturation, give an $R_{\mathrm{AA}}$ different from 1 . Final state effects, such as radiative and collisionial energy loss in the hot QCD matter, result in an $R_{\mathrm{AA}}$ smaller than unity [5].

\section{Total charm production cross section in pp}

Open charm production in inelastic pp collisions at a centre-of-mass energy $\sqrt{s}=2.76$ and $7 \mathrm{TeV}$ has been studied by the ALICE, ATLAS and LHCb experiment at the LHC. It is determined through the measurement of the direct reconstruction of D mesons in the hadronic decay channel [9-12]. In Fig. 1 (left panel), the total nucleon-nucleon charm cross sections are compared to results at lower collision energies and to next-to-leading-order (NLO) perturbative QCD calculations [13]. A very good agreement between the LHC experiments is observed. The data are systematically higher than the central value of the NLO pQCD calculations, but consistent within theoretical uncertainties. The pp measurements are important baselines for the Quarkonia measurements in heavy ion collisions 



Figure 2. Left panel: Nuclear modification factor $\left(R_{\mathrm{AA}}\right)$ of prompt $\mathrm{D}^{0}, \mathrm{D}^{+}, \mathrm{D}^{*+}$ and $\mathrm{D}_{\mathrm{s}}^{+}$mesons at mid-rapidity in the $0-7.5 \%$ centrality class of lead-lead collisions at $\sqrt{s_{\mathrm{NN}}}=2.76 \mathrm{TeV}$. Right panel: Transverse momentum dependence of the average $R_{\mathrm{AA}}$ of prompt $\mathrm{D}$ mesons at mid-rapidity in the $7.5 \%$ most central lead-lead collisions at $\sqrt{s_{\mathrm{NN}}}=2.76 \mathrm{TeV}$, compared to charged hadrons and pions.

and, of course, to test the parton spectra from pQCD calculations, used as an input for the energy loss models (see next section). One of the next challenges is to measure the total charm production cross section in lead-lead collisions, which requires the reconstruction of D mesons down to zero transverse momentum.

ATLAS has measured the $\mathrm{D}^{*+}$ meson production in jets in proton-proton collisions at $\sqrt{s}=7 \mathrm{TeV}$ for jets with transverse momentum between 25 and $70 \mathrm{GeV}$ in the pseudorapidity range $|\eta|<2.5$ [14]. Figure 1 (right panel) depicts the $\mathrm{D}^{*+}$ jet production rate $(R)$ for jets in the $p_{\mathrm{T}}$ range $25-30 \mathrm{GeV} / c$. The data are compared to state-of-the-art NLO pQCD calculations using the Monte Carlo event generators PYTHIA and HERWIG for parton showering. The predicted values of production rate by PYTHIA and POWHEG+PYTHIA are very similar, which is also the case when comparing calculations from HERWIG and POWHEG+HERWIG, as expected. Since $R$ is defined as the ratio between the number of $\mathrm{D}^{*+}$ jets and inclusive jets, the changes of total jet cross sections and $p_{\mathrm{T}}$ distributions between LO and NLO QCD calculations largely cancel. However, the Monte Carlo calculations fail to describe the data at small fractional momentum of the $\mathrm{D}^{*+}$ mesons $(\mathrm{z})$, and this discrepancy is strongest at low jet transverse momentum. The differences observed between the data and model predictions cannot be explained by varying the mixture of charm and beauty jets in the Monte Carlo calculations [14]. This is an indication that jet fragmentation into $\mathrm{D}^{*+}$ mesons is not well modeled in current Monte Carlo generators.

\section{Energy loss of prompt charmed mesons in $\mathrm{Pb}-\mathrm{Pb}$}

Heavy quarks (charm and beauty) are sensitive penetrating probes to study the transport properties of hot QCD matter. The general picture is that the scattered heavy quarks lose energy when traversing through the QCD matter by medium-induced gluon radiation and collisions with the light quarks in the medium. This interaction provides more insight on the energy loss mechanisms and thus on 

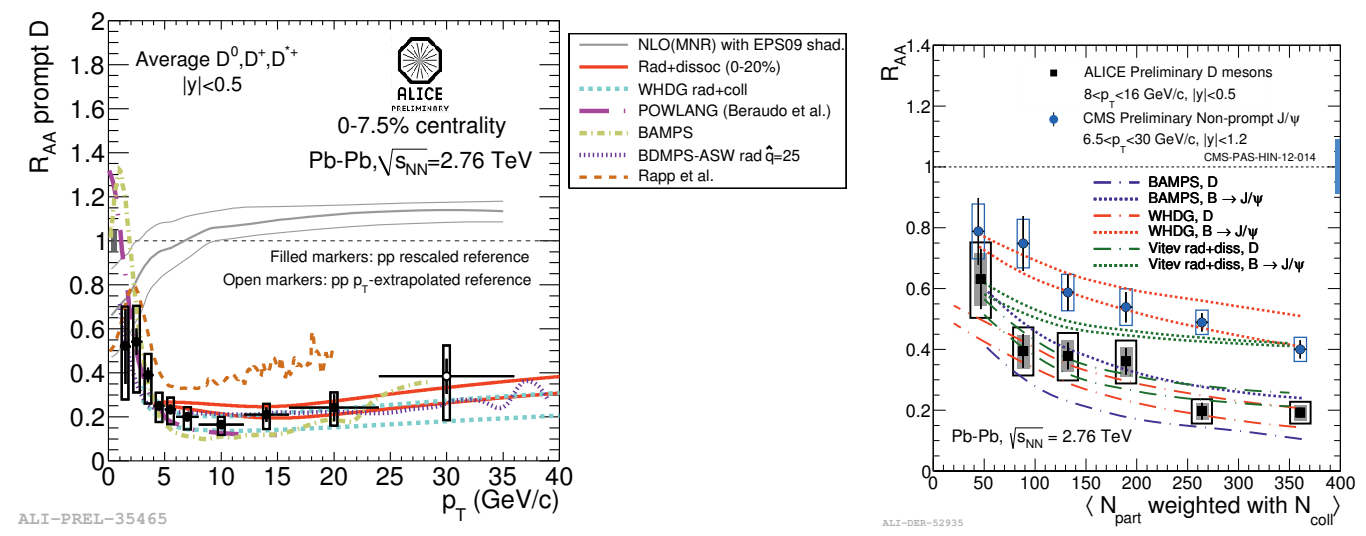

Figure 3. Left panel: Average $R_{\mathrm{AA}}$ of prompt D mesons, compared to next-to-leading-order (NLO) pQCD calculations with nuclear shadowing [13] and different parton energy-loss models [19]; Radiative and dissociation [20]; WHDG (collisional and radiative energy loss in anisotropic medium) [21]; POWLANG (collisional energy loss using Langevin approach) [22]; BAMPS (collisional energy loss in expanding medium) [23]; Collisional and LPM radiative energy loss [24]; BDMPS-ASW [25]; Collisional energy loss via D mesons resonances excitation and hydro evolution [26]. Right panel: Nuclear modification factor as a function of centrality for prompt $\mathrm{D}$ mesons measured by ALICE (black squares) and J/ $\Psi$ mesons from B decays from CMS [27].

dynamical properties of the QGP. Current theoretical developments were discussed at this conference by $[15,16]$. Recent results from the RHIC facility were reviewed by [6].

Theoretical models based on perturbative QCD predicted that heavy quarks should experience smaller energy loss than light quarks and gluons due to the suppression of gluon radiation at small angles (socalled dead-cone effect) $[17,18]$. Since this angle is mass dependent one expect less energy loss for charm hadrons at low transverse momentum compared to light quark hadrons (e.g. pions). By comparing the nuclear modification factor of charged pions $\left(R_{\mathrm{AA}}^{\pi^{ \pm}}\right)$, mostly originating from gluon fragmentation at this collision energy, with that of hadrons with charm $R_{\mathrm{AA}}^{\mathrm{D}}$ and beauty $R_{\mathrm{AA}}^{\mathrm{B}}$ the dependence of the energy loss on the parton nature (quark/gluon) and mass can be investigated. A hierarchy of suppression is expected with $R_{A A}^{\mathrm{B}}>R_{A A}^{\mathrm{D}}>R_{A A}^{\mathrm{h}^{ \pm}}$.

Figure 2 (left panel) illustrates the transverse momentum dependence of the $R_{\mathrm{AA}}$ for prompt $\mathrm{D}^{0}$, $\mathrm{D}^{+}, \mathrm{D}^{*+}$ and $\mathrm{D}_{\mathrm{s}}^{+}$mesons at mid-rapidity in the $7 \%$ most central lead-lead collisions at $\sqrt{s_{\mathrm{NN}}}=2.76$ $\mathrm{TeV}$ [19]. All D mesons show a strong suppression of their yield (factor of 4-5 at around $10 \mathrm{GeV} / \mathrm{c}$ ) and follow a similar trend at low $p_{\mathrm{T}}$, except the $\mathrm{D}_{\mathrm{s}}^{+}$mesons, which are less suppressed. The $\mathrm{D}_{\mathrm{s}}^{+}$ is of particular interest since it contains a charm and anti-strange quark. A higher production yield for $\mathrm{D}_{\mathrm{s}}^{+}$mesons at intermediate $p_{\mathrm{T}}$ is expected due to the enhancement of strangeness production and if it hadronizes via recombination in the medium. The average $\mathrm{D}$ meson $R_{\mathrm{AA}}$ is compared to charged hadrons and pions in Fig. 2 (right panel) in almost the same centrality bin and agrees within uncertainties. The current data suggest that prompt D mesons are suppressed at the same level as observed for light-quark hadrons, which was not expected due to the dead-cone and colour-charge effects. Data with higher precision are needed to test whether charm is less suppressed than light flavours at low $p_{\mathrm{T}}$.

Current theoretical model calculations [20-26] describe the observed suppression at high transverse momentum reasonably well [19] whereas the description at low transverse momentum $(\leq 2 \mathrm{GeV} / c)$ 



Figure 4. Average nuclear modification factor $\left(R_{\mathrm{pPb}}\right)$ of $\mathrm{D}$ mesons in $\mathrm{p}-\mathrm{Pb}$ collisions at $\sqrt{s_{\mathrm{NN}}}=5.023 \mathrm{TeV}$ (black circles) compared to (left panel) HVQMNR model calculations [13] using EPS09 set of parton distribution functions [29] and Color Glass Condensate-based calculation [30] and to (right panel) $\mathrm{Pb}-\mathrm{Pb}$ data at $\sqrt{s_{\mathrm{NN}}}=$ $2.76 \mathrm{TeV}$ (red triangles).

is more challenging (cf. Fig. 3, left panel). The centrality dependence of high- $p_{\mathrm{T}}$ prompt D mesons measured by ALICE [19] and J/ $\Psi$ mesons from B decays measured by CMS [27] are shown in Fig. 3 (right panel). The $\mathrm{D}$ and $\mathrm{B}$ mesons were selected in a compatible $p_{\mathrm{T}}$ range, namely $<p_{\mathrm{T}}^{\mathrm{B}}>\approx 11 \mathrm{GeV} / c$ and $\left\langle p_{\mathrm{T}}^{\mathrm{D}}>\approx 10 \mathrm{GeV} / c\right.$. The data indicate a larger suppression of $\mathrm{D}$ mesons in particular for the most central collisions. This is the first clear indication that in-medium parton energy loss decrease with increasing quark mass. However, further theoretical developments are needed to directly link these measurements to the drag diffusion coefficient of heavy quarks. This quantity was recently calculated in Lattice QCD [28].

\section{Open charm and cold nuclear matter effects}

To quantitatively understand the heavy ion data in terms of energy loss, it is important to disentangle hot nuclear matter effects from initial-state effects due to cold nuclear matter, such as the modification of the parton distribution functions in the nucleus [29] and saturation effects in the heavy flavour sector [30].

Initial-state effects can be investigated by measuring $\mathrm{D}$ production in $\mathrm{p}$ - $\mathrm{Pb}$ collisions. Recent results on light flavour production in $\mathrm{p}-\mathrm{Pb}$ collisions were present at this conference by [31]. The nuclear modification factor of the averaged prompt $\mathrm{D}^{0}, \mathrm{D}^{+}$and $\mathrm{D}^{*+}$ mesons in minimum bias $\mathrm{p}-\mathrm{Pb}$ at $\sqrt{s_{\mathrm{NN}}}=$ 5.023 TeV is depicted in Fig. 4 (left panel). The $R_{\mathrm{AA}}$ is compatible with unity within systematic uncertainties over the full $p_{\mathrm{T}}$ range. The data are compared with $\mathrm{pQCD}$ calculations based on the HVQMNR model calculations [13] combined with the EPS09 modifications of the parton distribution functions [29] and with a Color Glass Condensate-based calculation [30]. Both theoretical models describe the data within experimental uncertainties. Thus, the strong suppression of the heavy flavour hadron yield observed in central $\mathrm{Pb}-\mathrm{Pb}$ collisions is indeed a final-state effect (cf. Fig. 4, right panel), arising from the hot QCD matter. 

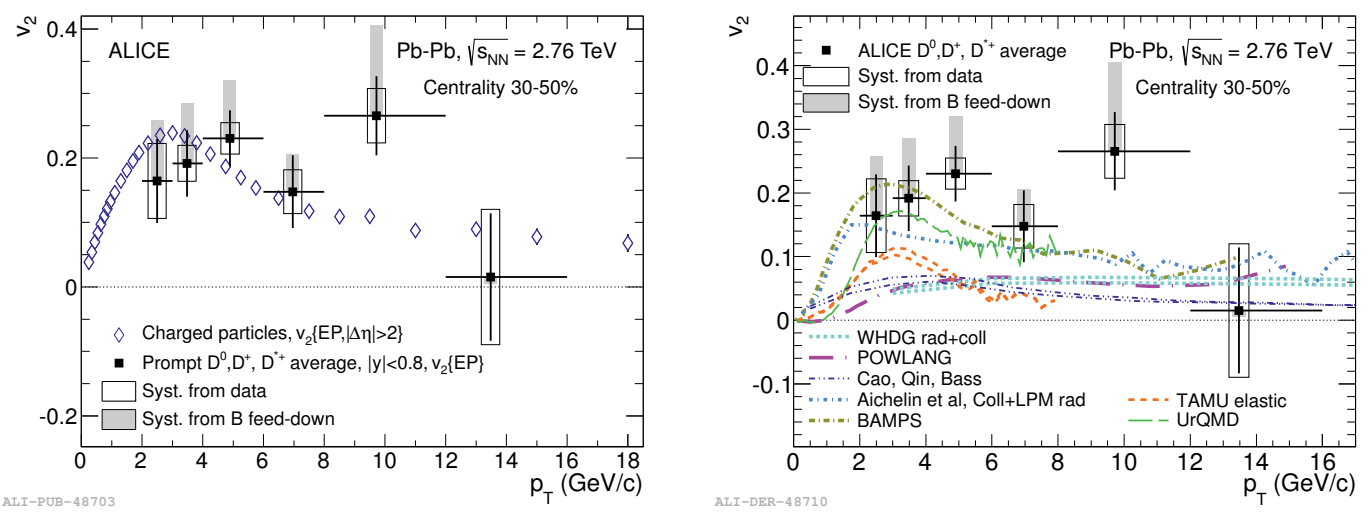

Figure 5. Transverse momentum dependence of the elliptic flow coefficient $v_{2}$ of $\mathrm{D}$ mesons for the centrality class $30-50 \%$ (full black squares) [32], compard to (left panel) data for charged hadrons (blue open triangles) and (left panel) theoretical model calculations [20-26].

\section{Azimuthal anisotropy of prompt $\mathrm{D}$ mesons in $\mathrm{Pb}-\mathrm{Pb}$}

Measurements of the momentum distribution of emitted particles and comparison with hydro-dynamic model calculations have shown that the outward steaming particles move collectively, with the patterns arising from variations of pressure gradients early after the collision. This phenomenon is called azimuthal anisotropy or elliptic flow and is analogous to the properties of fluid motion. The study of the azimuthal anisotropy of heavy-quark particles is particularly interesting as it provides information on the degree of thermalisation of heavy quarks in the medium.

The transverse momentum dependence of the elliptic flow coefficient $v_{2}$ of D mesons [32] is depicted in Fig. 5 (left panel) together with that of inclusive charged hadrons for the centrality interval 30-50\%. Despite the large uncertainties, the $v_{2}$ measurement of charmed mesons shows a sizable anisotropy (3$5 \sigma$ ), suggesting that charm quarks may take part in the collective motion generated at the quark level in the deconfined stage. The $v_{2}$ results are confronted with theoretical model calculations in Fig. 5 (right panel). The models describe the features of the data reasonably well, but a quantitative description of both energy loss and elliptic flow remains challenging for them.

\section{Heavy flavour decay lepton $R_{\mathrm{AA}}$ and $v_{2}$}

The semi-leptonic decay of $\mathrm{D}$ and $\mathrm{B}$ mesons, with a branching ratio of about $10 \%$, also provides an access to study open charm and beauty production. The transverse momentum dependence of the heavy flavour decay electron $R_{\mathrm{AA}}$ and $v_{2}$ at midrapidity in lead-lead collisions at $\sqrt{s_{\mathrm{NN}}}=2.76 \mathrm{TeV}$ in the centrality range $0-10 \%$ and $20-40 \%$, respectively, by ALICE are shown in Fig. 6. Muons from heavy-flavour hadron decays at forward rapidity $(-4<\eta<-2.5)$ have, within uncertainties, a similar $R_{\mathrm{AA}}$ as single electrons in the $p_{\mathrm{T}}$ range $4-10 \mathrm{GeV} / c$ (not shown in Fig. 6). Both single electrons and muons are suppressed to the same level as observed for prompt $\mathrm{D}$ mesons for $3<p_{\mathrm{T}}<7 \mathrm{GeV} / c$, giving a clear indication for substantial energy loss of heavy quarks in the medium. The single electron $R_{\mathrm{AA}}$ is higher than the prompt charmed meson $R_{\mathrm{AA}}$ for $p_{\mathrm{T}}>7 \mathrm{GeV} / c$ due to the beauty contribution. ATLAS measured the centrality dependence of the $R_{\mathrm{CP}}$ of single muons at midrapidity. The $R_{\mathrm{CP}}$ decreases continuously from low to high centralities and reaches $0.4-0.5$ at $\left\langle N_{\text {part }}\right\rangle=350$ [33]. 

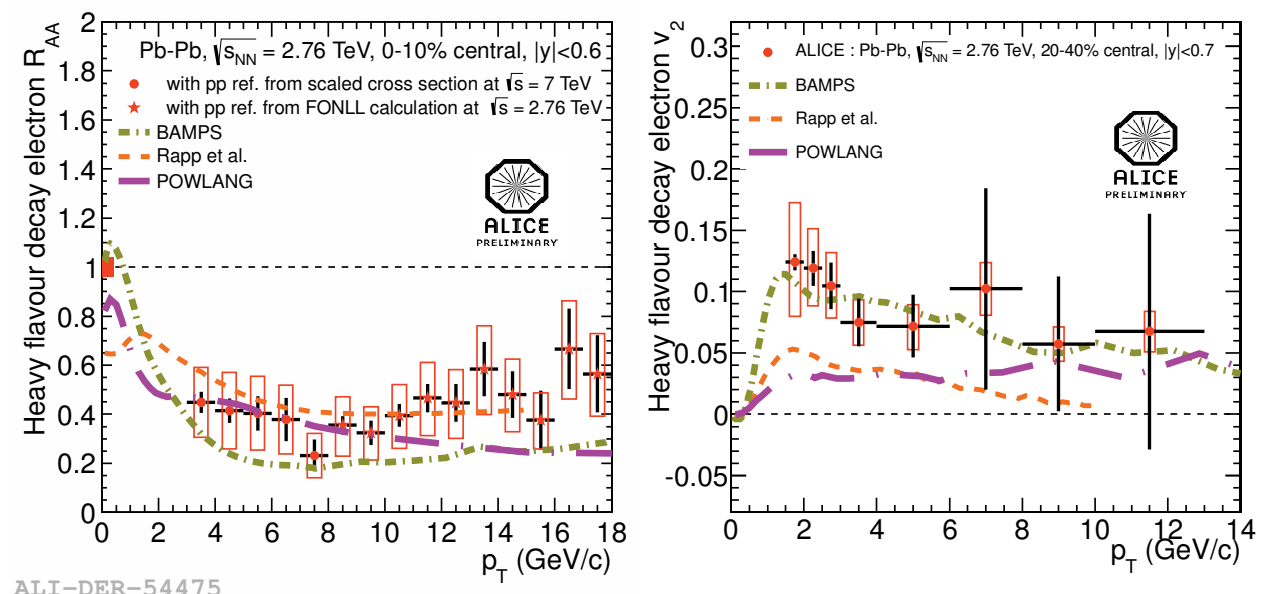

Figure 6. Transverse momentum dependence of the heavy flavour decay electron $R_{\mathrm{AA}}$ (left panel) and $v_{2}$ (right panel) at midrapidity in lead-lead collisions at $\sqrt{s_{\mathrm{NN}}}=2.76 \mathrm{TeV}$ in the centrality range $0-10 \%$ and $20-40 \%$, respectively. The data are compared with different theoretical model calculations [21, 23, 26].

The non-zero $v_{2}$ observed in the $20-40 \%$ central lead-lead events suggests that heavy quarks experience strong re-interactions within the medium, as for prompt $\mathrm{D}$ mesons.

For the first time the LHC allows more differential, precision measurements of heavy quark production ( $R_{\mathrm{AA}}, v_{2}$, its centrality dependence and heavy flavour particle correlations) in heavy ion collisions. As illustrated in Fig. 6, a simultaneous description of the nuclear modification factor and elliptic flow coefficient for heavy-flavour particles is challenging for current theoretical model calculations [21, $23,26]$.

\section{Heavy-quark particle correlations in $\mathrm{Pb}-\mathrm{Pb}$}

Correlations between heavy quarks are largely not effected by the fragmentation process in protonproton interactions. In heavy-ion collisions, however, the medium alters the fragmentation process, so that observables are sensitive to the properties of the medium [34, 35]. It has been shown that the fragmentation function, which describes how the parton momentum is distributed among the finalstate hadrons, is most suited for these detailed studies. Flavour conservation implies that heavy quarks are produced in pairs. Momentum conservation requires that these pairs are correlated in relative azimuth $(\Delta \phi)$ in the plane perpendicular to the colliding beams. Since heavy quarks are produced in $2 \rightarrow 2(g g \rightarrow Q \bar{Q})$ and $2 \rightarrow 3$ (e.g. $g g \rightarrow Q \bar{Q} g$ ) processes [13], the azimuthal correlation is not strictly back-to-back.

One method of exploiting this pair production characteristic is to measure the azimuthal angular correlation $(\Delta \phi)$ of electrons from semileptonic decays of heavy-flavor hadrons with charged hadrons. Figure 7 (left panel) shows the $\Delta \phi$ distribution measured by ALICE [36], which exhibits a distinct near-side $(-\pi / 2<\Delta \phi<\pi / 2)$ correlation peak. The ratio of the measured correlation yield in leadlead collisions relative to the one in pp interactions, $I_{\mathrm{AA}}$,

$$
I_{\mathrm{AA}}=\frac{\int_{\phi_{1}}^{\phi_{2}} d \Delta \phi\left(d N_{A A} / d \Delta \phi\right)}{\int_{\phi_{1}}^{\phi_{2}} d \Delta \phi\left(d N_{p p} / d \Delta \phi\right)},
$$



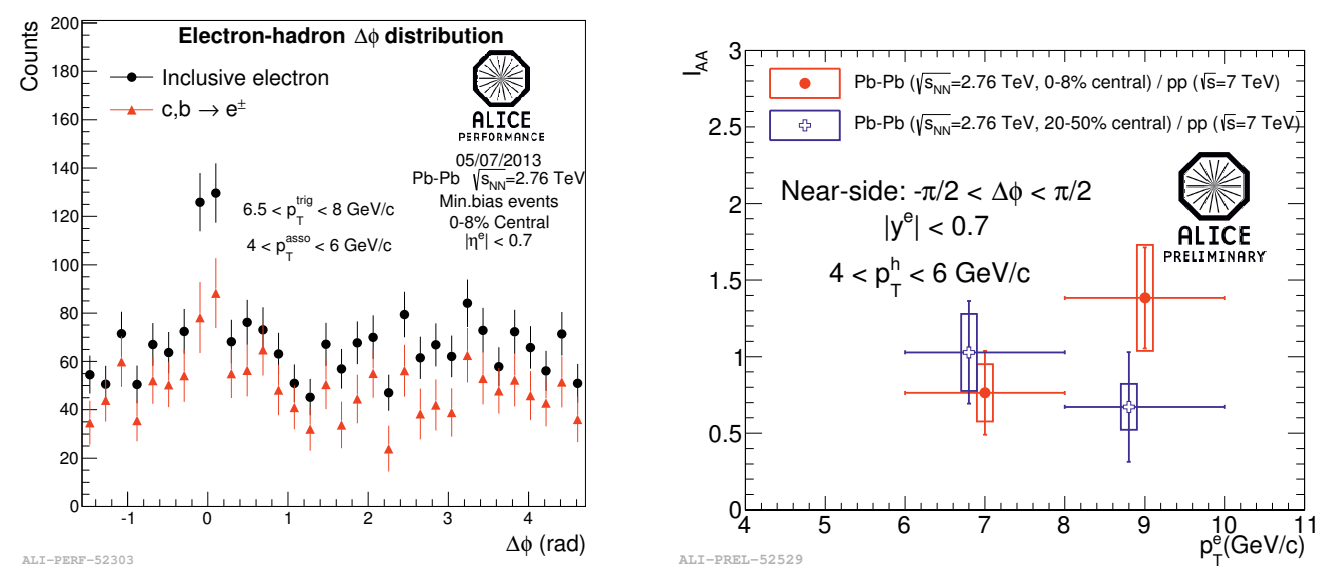

Figure 7. Left panel: Azimuthal angular correlations $(\Delta \phi)$ between inclusive (full black circles) and heavyflavor decay electrons (full red triangles) at midrapidity in the $8 \%$ most central $\mathrm{Pb}-\mathrm{Pb}$ collisions at $\sqrt{s_{\mathrm{NN}}}=$ $2.76 \mathrm{TeV}$. Right panel: Single electron transverse momentum dependence of the $I_{A A}$ of the near-side correlation peak in $\mathrm{Pb}-\mathrm{Pb}$ collisions in the centrality intervals $0-8 \%$ (full red circles) and $20-50 \%$ (open blue crosses) [36]. The associated charged hadron $p_{\mathrm{T}}$ range is $4-6 \mathrm{GeV} / c$. The near-side correlation yield in lead-lead collisions is normalized to the one in $7 \mathrm{TeV}$ pp interactions.

on the near side as a the function of electron trigger $p_{\mathrm{T}}$ is shown in Fig. 7 (right panel). An excess, $I_{\mathrm{AA}}>1$, may be expected at high electron $p_{\mathrm{T}}$ in central collisions due to the depletion and broadening of the correlation signal in the medium. These results agree with previous measurements at RHIC [37]. However, so far they are statistics limited and more precision data is needed, both at RHIC and the LHC, to draw final conclusions. The lead-lead data from LHC run2 might be sufficient to finalize these important studies.

\section{Upgrades}

From the current heavy flavour data and detector performance it became evident that higher precision is necessary to determine the properties of strongly interacting matter at the LHC. Therefore the LHC experiments setup a programme of detector upgrades to be installed in the shutdown planned for 2018/2019, to address these experimental challenges [38]. Especially important for the open charm measurements are an improved secondary vertex resolution and enhanced rate capabilities to allow $\mathrm{Pb}-\mathrm{Pb}$ data taking at higher interaction rates.

\section{Summary}

In summary, recent results on the nuclear modification factor $\left(R_{\mathrm{AA}}\right)$ and elliptic flow coefficient $\left(v_{2}\right)$ of prompt charmed mesons and heavy-flavour decay electrons at mid-rapidity and single muons from heavy-flavour hadron decays at forward rapidity $(-4<|\eta|<-2.5)$ were presented.

A positive elliptic flow coefficient was measured for both prompt D mesons and heavy flavour decay electrons, suggesting that heavy quarks may take part in the collective motion built up at the quark level in the deconfined stage. 
The centrality dependence of the $R_{\mathrm{AA}}$ for prompt $\mathrm{D}$ mesons, measured in the transverse momentum range $8<p_{\mathrm{T}}<16 \mathrm{GeV} / c$, and $J / \Psi$ mesons from $\mathrm{B}$ decays shows a clear indication for a different suppression of the production yields, in agreement with the expected quark mass dependence of the parton energy loss.

The nuclear modification factor of heavy-flavour decay electrons and prompt charmed mesons in $\mathrm{p}$ $\mathrm{Pb}$ collisions at $\sqrt{s_{\mathrm{NN}}}=5.023 \mathrm{TeV}$ is compatible with unity in the measured transverse momentum range. These measurements confirm the assumption that initial-state effects are small at sufficient high $p_{\mathrm{T}}$. Thus the large suppression of the heavy-flavour hadron yield observed in $\mathrm{Pb}-\mathrm{Pb}$ collisions is a final-state effect and arises from interactions of heavy quarks with the hot QCD matter.

Heavy-flavour particle correlations will allow measuring the possible modification of the fragmentation function of heavy quarks in ultra-relativistic heavy ion collisions.

Indication have been found that jet fragmentation into charmed mesons in elementary proton-proton interactions is not well modeled in current Monte Carlo generators.

\section{Acknowledgments}

I would like to thank the organizers for the invitation and the inspiring atmosphere at the conference. Sincerest thanks to the ALICE, ATLAS and CMS Collaboration for providing the data and the LHC accelerator team.

The European Research Council has provided financial support under the European Community's Seventh Framework Programme (FP7/2007-2013) / ERC grant agreement no 210223. This work was also supported by a Vidi grant from the Netherlands Organisation for Scientific Research (project number 680-47-232) and Projectruimte grants from the Dutch Foundation for Fundamental Research (project numbers: 10PR2884 and 12PR3083).

\section{References}

[1] P. Braun-Munzinger, these proceedings.

[2] U. Wiedemann, these proceedings.

[3] The CERN Large Hadron Collider: Accelerator and Experiments, Vol. 1 and 2, Edited by A. Breskin and R. Voss, JINST 3, S08001-S08007 (2008).

[4] H. Satz, these proceedings.

[5] P. di Nezza, these proceedings.

[6] J. Bielcikova, these proceedings.

[7] B. Müller, Nucl. Phys. A 750, 84 (2005).

[8] J. Uphoff, O. Fochler, Z. Xu and C. Greiner, Phys. Rev. C 82, 044906 (2010).

[9] B. Abelev et al. (ALICE Collaboration), JHEP 07, 191 (2012).

[10] B. Abelev et al. (ALICE Collaboration), JHEP 01, 128 (2012).

[11] ATLAS Collaboration, ATLAS-PHYS-PUB-2011-012 (2011) and ATLAS-CONF-2011-017 (2011).

[12] LHCb Collaboration, LHCb-CONF-2010-013 (2010).

[13] M. L. Mangano, P. Nason and G. Ridolfi, Nucl. Phys. B 373, 295 (1992).

[14] G. Aad et al. (ATLAS Collaboration), Phys. Rev. D 85, 052005 (2012).

[15] M. Nardi, these proceedings.

[16] P.-B. Gaussiaux, these proceedings.

[17] Y. Dokshitzer, D. Kharzeev, Phys. Lett. B 519, 199 (2001), hep-ph/0106202. 


\section{ICNFP 2013}

[18] S. Wicks, W. Horowitz, M. Djordjevic and M. Gyulassy, Nucl. Phys. A 784, 426 (2007).

[19] B. Abelev et al. (ALICE Collaboration), JHEP 09, 112 (2012).

[20] R. Sharma, I. Vitev and B. W. Zhang, Phys. Rev. C80 (2009) 054902 and Y. He, I. Vitev and B. W. Zhang, Phys. Lett. B 713, 224 (2012).

[21] W. A. Horowitz and M. Gyulassy, J. Phys. G38, 124114 (2011).

[22] W. M. Alberico et al., Eur. Phyis J. C 71, 1666 (2011).

[23] O. Fochler, J. Uphoff, Z. Xu and C. Greiner, J. Phys. G38, 124152 (2011).

[24] J. Aichelin et al., Phys. Rev. C79, 044906 (2009).

[25] N. Armesto, A. Dainese, C. A. Salgado and U. A. Wiedemann, Phys. Rev. D71, 054027 (2005).

[26] M. He, R. J. Fries and R. Rapp, Phys. Rev. Lett. 110, 112301 (2013).

[27] S. Chatrchyan et al. (CMS Collaboration) 2012 CMS-PAS-HIN-12-014.

[28] D. Banerjee, S. Datta, R. Gavai and P. Majumdar, Phys. Rev. D 85, 014510 (2012).

[29] K. J. Eskola H. Paukkunen and C. A. Salgado, JHEP 0904, 065 (2009).

[30] H. Fujii and K. Watanabe, Nucl Phys. A 920, 78 (2013).

[31] L. Ramello, these proceedings.

[32] B. Abelev et al. (ALICE Collaboration), Phys. Rev. Lett. 111, 102301 (2013).

[33] ATLAS Collaboration, ATLAS-CONF-2012-050, 26 May 2012.

[34] M. Nahrgang, J. Aichelin, P. B. Gossiaux and K. Werner, arXiv: 1305.3823.

[35] S. Cao, G.-Y. Qin, S. A. Bass and B. Müller, J. Phys.: Conf. Ser. 446, 012035 (2013).

[36] D. Thomas (ALICE Collaboration), Proceedings of the Strange Quark Matter conference, arXiv:1312.1489.

[37] A. Adare et al. (PHENIX Collaboration), Phys. Rev. C 83, 044912 (2011).

[38] T. Peitzmann, these proceedings. 